\section{Disparidades étnico-raciais em saúde autoavaliada: análise multinível de 2.697 indivíduos residentes em 145 municípios brasileiros}

\author{
Racial/ethnic disparities in self-rated health: \\ a multilevel analysis of 2,697 individuals \\ in 145 Brazilian municipalities
}

Disparidades etno-raciales en la autopercepción de salud: un análisis multinivel de 2.697 personas que viven en 145 municipios brasileños
Alexandre Dias Porto Chiavegatto Filho 1 Ruy Laurenti ${ }^{1}$

\section{Resumo}

Recent literature reviews have shown that studies analyzing racial/ethnic disparities in Brazil are still scarce. Multilevel approaches are necessary, since race is a socially constructed concept and can vary by area of residence. The analysis included 2,697 individuals from 145 Brazilian municipalities (counties), classified by race (white, black, or mixed). Multilevel models were fitted using Bayesian inference with Markov Chain Monte Carlo methods. After including demographic, socioeconomic, and health access variables, black and mixed-race individuals showed higher odds of negative self-rated health $(O R=1.71 ; 95 \% C I: 1.24 ; 2.37$ and $O R=1.37$; 95\%CI: 1.10; 1.71 , respectively). Characteristics of the area of residence did not significantly affect the association between race and self-rated health. Racial/ethnic disparities lost their statistical significance after re-categorization of the dependent variable. The results indicate that racial/ethnic disparities in health in Brazil may be deeper and more complex than expected.

Ethnicity and Health; Multilevel Analysis; Socioeconomic Factors
Revisões recentes da literatura indicam que o número de estudos sobre disparidades étnicoraciais no Brasil é escasso. A análise multinível torna-se necessária já que o conceito de raça/cor é socialmente construído e pode variar segundo local de residência. Foram analisados 2.697 indivíduos residentes em 145 municípios brasileiros, segundo raça (branca, preta e parda). Foram ajustados modelos multinível utilizando inferência bayesiana pelo método Monte Carlo via Cadeias de Markov. Após a inclusão de variáveis demográficas, socioeconômicas e de acesso a serviços de saúde, indivíduos de raça preta e parda tiveram maior razão de chances de avaliarem sua saúde como negativa $(R C=1,71 ;$ IC95\%: 1,24; 2,37 e $R C=1,37$; IC95\%: 1,10; 1,71, respectivamente). Características do local de residência não alteraram significativamente a relação entre raça/cor e saúde autoavaliada. Após a recategorização da variável dependente, as características étnico-raciais perderam significância estatística. O presente estudo indica que as disparidades raciais em saúde podem ser mais complexas do que o esperado.

Origem Étnica e Saúde; Análise Multinível; Fatores Socioeconômicos 


\section{Introdução}

Estudos sobre características étnico-raciais são frequentes na maioria dos países com heterogeneidade racial, principalmente nos Estados Unidos, onde as disparidades raciais em saúde têm sido detalhadamente monitoradas por pelo menos cinco décadas 1 . Análises recentes da literatura têm concluído que esse não é o caso do Brasil 2,3. Segundo Araújo et al. 4, apesar do notável aumento recente, a produção brasileira sobre o tema ainda pode ser considerada ínfima se comparada com países desenvolvidos.

De acordo com Bailey 5, a origem do baixo interesse pela análise de disparidades étnicoraciais pode ser atribuída à teoria de democracia racial, conceito originado na metade do século XX que defendia que o Brasil era dotado de boas relações raciais devido ao seu histórico de miscigenação e definições raciais imprecisas. Internacionalmente, o Brasil era frequentemente apontado como contraposto e um exemplo a ser seguido para países segregacionistas como a África do Sul e os Estados Unidos 6. Segundo Telles 7, a ideia da existência de uma democracia racial se incorporou na ideologia nacional e denunciar a existência de racismo passou a ser visto como uma atitude antibrasileira.

A inclusão de uma questão sobre diferenças atribuídas à raça, cor e/ou etnia é um processo recente para a maioria das pesquisas nacionais. A sua presença nas estatísticas vitais (declaração de óbito e de nascidos vivos) foi estabelecida por um decreto de $1996{ }^{8}$, sendo apenas incluída de fato a partir de 1998. No censo seguinte, de 2000, não houve questão sobre raça/cor no questionário completo aplicado a toda a população, estando presente somente no questionário amostral.

A definição de categorias para a identificação étnico-racial é um tema complexo, que tem sido extensivamente debatido no Brasil durante as últimas décadas 9,10,11. Conceitualmente, raça diz respeito às características fenotípicas do indivíduo, com destaque para a cor da pele. Por outro lado, a questão da etnia está fortemente relacionada a características socioculturais. Estudos mostram que, ao avaliar a sua própria raça, indivíduos levam em consideração tanto características físicas pessoais, quanto o contexto social em que vivem 12 , justificando o uso do termo "características étnico-raciais". A categorização étnico-racial adotada pelo presente estudo é a utilizada pelo questionário de autoavaliação do censo, no qual os indivíduos se classificam em cinco categorias: branca, preta, parda, amarela e indígena.

Análises recentes mostram que a ausência de um debate aberto sobre as disparidades étnico- raciais não tem evitado o problema. Segundo o Censo Demográfico de 2010 (Instituto Brasileiro de Geografia e Estatística. Sistema IBGE de Recuperação Automática. http://www.sidra.ibge. gov.br, acessado em 10/Jan/2012), brancos têm renda $84,5 \%$ maior que pretos e $81,95 \%$ maior que pardos (R\$ 1.535,47, R\$ 832,25 e R\$ 843,87, respectivamente). Indivíduos de raça branca têm também maior taxa de alfabetização $(92,78 \%) \mathrm{em}$ relação a pretos $(81,66 \%)$ e pardos $(82,85 \%)$.

Estudos brasileiros têm apontado para uma associação entre características étnico-raciais e saúde em determinados contextos. Uma análise realizada no Mato Grosso do Sul verificou uma razão de chances para morte materna 5,13 vezes maior para mulheres classificadas como sendo de raça preta em relação a mulheres consideradas brancas 13. A mortalidade infantil também é mais importante para recém-nascidos considerados de raça preta, sendo de $30 \%$ a $80 \%$ maior do que entre os considerados como brancos 14 . O acesso aos serviços de saúde é diferente segundo raça, sendo a probabilidade de mulheres consideradas negras realizarem exames de detecção de câncer de mama consideravelmente menor em relação a brancas 15 .

Uma possibilidade de medir a situação de saúde como um todo é a análise da autoavaliação do estado de saúde, que diversos estudos têm validado como um indicador de mortalidade futura 16,17. Analisando essa variável, estudos recentes têm apresentado resultados conflitantes. Uma análise utilizando dados da Pesquisa Nacional por Amostra de Domicílios (PNAD) verificou que a razão de chances de brancos e negros declararem sua saúde como muito boa ou boa não foi significativa em 1998 e apenas marginalmente significativa em 2003 (para pardos foi significativamente pior nos dois períodos) 18 . Um outro estudo utilizando dados da PNAD de 1998 verificou que as diferenças raciais não foram significativas após a inclusão das variáveis renda e educação 19. Por outro lado, Barata et al. 20, também utilizando dados da PNAD, verificaram diferenças significativas na saúde autoavaliada para pretos e pardos (analisados em conjunto) em relação a brancos.

Uma metodologia ainda pouco utilizada em estudos nacionais sobre disparidades étnicoraciais é a abordagem multinível 21, que permite a introdução de dois ou mais níveis na análise, frequentemente um individual e pelo menos um contextual. Análises internacionais têm apontado para importância das características do local de residência para a associação entre raça e nível de saúde autodeclarado, um efeito complementar à influência de características individuais. Um estudo realizado na Inglaterra encontrou que uma pior situação socioeconômica local 
teve maior efeito na saúde de indivíduos de raça branca ${ }^{22}$. Uma análise de regiões metropolitanas dos Estados Unidos verificou que uma maior densidade local de negros estava associada a uma maior probabilidade de homens e mulheres negras declararem saúde ruim 24.

A inclusão de uma abordagem multinível é particularmente importante para o caso da análise de disparidades étnico-raciais já que raça é uma construção social. No caso do Brasil, um país com presença histórica de miscigenação, é possível um mesmo indivíduo se considerar branco em um determinado ambiente socioeconômico e pardo (ou mesmo preto) em outro 24.

Uma outra questão importante na literatura recente sobre raça/cor é determinar se as desigualdades étnico-raciais em saúde têm relevância como fator independente, ou se são apenas uma consequência dos piores indicadores socioeconômicos apresentados por negros em relação a brancos 12. Segundo Travassos \& Williams 3, a presença de forte associação entre condição socioeconômica e classificação étnico-racial pode explicar parte das disparidades encontradas em estudos da área. Uma revisão recente de estudos étnico-raciais, verificou que somente $27 \%$ dos artigos incluíram fatores socioeconômicos em seus modelos estatísticos 10 .

O objetivo do presente estudo é primeiramente testar a existência de diferenças étnicoraciais na autoavaliação de saúde controlando-se por fatores socioeconômicos, demográficos e de acesso a serviços de saúde. Pretende-se também analisar se características do local de residência dos indivíduos explicam parte dessa associação.

\section{Metodologia}

Foram analisados os resultados do Sistema de Indicadores de Percepção Social (SIPS), primeira edição, realizado pelo Instituto de Pesquisa Econômica Aplicada (IPEA). Participantes foram selecionados pela técnica de amostragem por cotas, com o objetivo de garantir representatividade com nível de $95 \%$ de confiança para as regiões brasileiras 25 . Os parâmetros para a definição de representatividade vieram da $\mathrm{PNAD} / 2008$ e foram estabelecidos pela distribuição das variáveis sexo, faixa etária, faixa de renda e escolaridade 26 .

O questionário inicial foi aplicado a uma amostra de 2.773 pessoas residentes em domicílios particulares permanentes de 3 a 19 de novembro de 2010. A pesquisa incluiu todas as Unidades de Federação (UF) brasileiras, com média de 102,70 indivíduos por UF, e 145 municípios, com média de 18,99 indivíduos por município. Para a questão étnico-racial foi utilizado o crité- rio de autoavaliação de raça/cor, onde indivíduos selecionaram uma das seguintes categorias: branca, preta, amarela, parda, indígena e não sabe/não responde. Do total de indivíduos analisados, 19 (0,69\%) não informaram situação de saúde e sete $(0,25 \%)$ não informaram raça/cor e foram excluídos da análise. Indivíduos de raça/cor amarela e indígena compuseram apenas $1,55 \%$ e $0,25 \%$ da amostra e também foram excluídos. Isso totalizou 2.697 indivíduos efetivamente analisados pelo presente estudo.

Foi utilizada a autoavaliação de saúde como variável resposta, analisada pela questão: "De um modo geral, você considera seu próprio estado de saúde como: (1) muito bom, (2) bom, (3) regular, (4) ruim, (5) muito ruim". Foi utilizada a definição aplicada pela maioria dos estudos de autoavaliação de saúde 27 , onde as categorias "muito bom" e "bom" são agregadas em um mesmo grupo (autoavaliação positiva), e as categorias "regular", "ruim" e "muito ruim" em outro (autoavaliação negativa). Além de permitir a comparação com outros estudos, a inclusão da categoria "regular" junto com "ruim" e "muito ruim" se justifica pela sua forte associação com maior probabilidade de mortalidade futura 28 . Como teste de sensibilidade, os modelos completos foram também estimados agregando a categoria "regular" junto com as positivas. Foram incluídas no modelo variáveis referentes a características demográficas (sexo e idade), socioeconômicas (renda e escolaridade), conjugal (estado civil), acesso a serviços de saúde (presença de seguro privado de saúde e cadastro do domicílio no Programa Saúde da Família - PSF) e características do estado e do município de residência (percentual de pretos e pardos, taxa de alfabetização e renda nominal média). As variáveis contextuais (referentes aos estados e municípios) foram calculadas utilizando os resultados do censo de 2010.

\section{Análise estatística}

Os parâmetros dos modelos foram estimados utilizando a metodologia multinível (também conhecida como modelagem mista ou hierarquizada). Diversos benefícios têm sido apontados para o uso dessa metodologia em estudos epidemiológicos, como a possibilidade de controlar pela presença de não-independência de observações aglomeradas (no caso, indivíduos dentro de municípios situados dentro de estados) e o controle tanto dos efeitos da falácia ecológica (onde resultados agregados não refletem características individuais) como da falácia atomística (quando estudos de indivíduos são utilizados inadequadamente para inferir risco populacional) 29,30,31. Para o presente estudo, o uso da abordagem 
multinível tem também como objetivo analisar a existência de um efeito contextual do município e do estado de residência na autoavaliação de saúde segundo raças. Foram incluídos três níveis no modelo: individual (nível 1), municipal (nível 2) e estadual (nível 3), permitindo uma análise de significância estatística da variância para dois níveis (municipal e estadual).

Foi utilizada inferência bayesiana (pelo uso de métodos de Monte Carlo via Cadeias de Markov) para estimar os parâmetros do modelo devido à natureza dicotômica da variável resposta (autoavaliação de saúde). Em modelos logísticos binários, a abordagem bayesiana é recomendada para diminuir o viés inerente ao uso de procedimentos de máxima-verossimilhança em análises multinível ${ }^{32}$. Além disso, permite a comparação da adequação dos modelos testados, pelo cálculo do coeficiente de deviance information criterion (DIC), uma análise de badness-of-fit (ou seja, de falta de qualidade de ajuste), onde valores maiores indicam pior adequação do modelo em relação à variável resposta 33 . Os parâmetros dos modelos multinível foram apresentados nas tabelas em termos de razão de chances para facilitar a interpretação.

Foi utilizado o software MLwiN 2.25 (Centre for Multilevel Modelling, Bristol, Reino Unido), especializado em análise multinível, para a estimação dos parâmetros.

\section{Resultados}

A Tabela 1 apresenta a distribuição das características individuais, para o total e para aqueles com autoavaliação negativa do nível de saúde. Entre os indivíduos incluídos na análise, 32,37\% avaliaram sua situação de saúde como negativa. Brancos tiveram menor proporção de autoavaliação negativa (27,75\%), em comparação com pretos $(40,07 \%)$ e pardos $(34,97 \%)$. Mulheres, indivíduos sem seguro privado de saúde e residentes de domicílios cadastrados no PSF apresentaram maior proporção de autoavaliação negativa. Pela análise das variáveis socioeconômicas é possível verificar um gradiente crescente, onde indivíduos com maior escolaridade e maior renda apresentaram menor proporção de autoavaliação negativa para todas as categorias.

Em relação à idade, única variável contínua da análise, a mediana para o total de indivíduos foi de 39 anos, aumentando para 49 entre os indivíduos com autoavaliação negativa de saúde. Entre as variáveis contextuais referentes ao nível municipal, a mediana do percentual de pretos e pardos foi $55,58 \%$, a taxa de alfabetização de $94,6 \%$ e a renda nominal média $\mathrm{R} \$ 2.004,98$.
Entre os estados, as medianas foram respectivamente $61,79 \%, 90,4 \%$ e $\mathrm{R} \$ 1.843,49$.

A Tabela 2 apresenta os resultados da análise multinível para as características individuais. No modelo nulo é possível verificar uma variância estatisticamente significativa ao nível estadual (0,224; IC95\%: 0,014; 0,434), mas não ao nível municipal $(0,027$; IC95\%: -0,018; 0,072). No modelo 1 foi incluída a variável étnico-racial. Tanto pretos quanto pardos tiveram maior razão de chances, em relação a brancos, de autoavaliar sua saúde como negativa (1,61 e 1,32, respectivamente), e a variância ao nível estadual manteve-se estatisticamente significativa (0,193; IC95\%: 0,005; 0,381). O modelo 2 teve a inclusão das características demográficas (sexo e idade). Ambas foram estatisticamente significativas $(\mathrm{p}<0,05)$, assim como as duas categorias de raça/cor e a variância ao nível estadual. No modelo 3 foram incluídas as características socioeconômicas (renda e escolaridade). Todas as categorias foram estatisticamente significativas $(\mathrm{p}<0,05)$, mas a variância ao nível estadual perdeu significância. O modelo 4 teve a inclusão do estado civil, que não apresentou significância estatística para nenhuma das suas três categorias. Além disso, não teve efeito considerável nas outras variáveis, sendo portanto excluída dos modelos futuros. É importante verificar que para todos os modelos houve diminuição do DIC, indicando melhor adequação do modelo. A melhoria em relação ao último modelo foi quase nula, justificando a sua exclusão.

A Tabela 3 apresenta os modelos com a inclusão de variáveis referente ao acesso a serviços de saúde e características do local de residência. No modelo 5 foi incluída a presença de seguro saúde e cadastro do domicílio no PSF. A primeira foi estatisticamente significativa $(\mathrm{RC}=1,45$; IC95\%: $1,14 ; 1,85)$ enquanto a segunda não $(\mathrm{RC}=0,86$; IC95\%: 0,70; 1,06), sendo esta excluída dos modelos seguintes. As outras variáveis desse modelo mantiveram significância, menos a categoria de renda de 5 a 10 salários mínimos $(\mathrm{OR}=0,79$; IC95\%: 0,59; 1,07). No modelo 6 foram incluídas características do município de residência. As três variáveis (percentual de pretos e pardos, taxa de alfabetização e renda nominal média) tiveram pouco ou nenhum efeito nos parâmetros das características individuais. A única que pode ser considerada significativa é a taxa de alfabetização, apesar de apenas marginalmente $(\mathrm{RC}=0,98$; IC95\%: 0,96; 0,99). Resultados semelhantes foram verificados para o modelo 7 , que teve a inclusão de características do estado de residência. O modelo 5, que incluiu características individuais e acesso a serviços de saúde, mas não características do local de residência, foi o que apresentou menor DIC, indicando melhor ajuste. 
Tabela 1

Distribuição das características individuais da amostra. Brasil, 2010

\begin{tabular}{|c|c|c|c|c|}
\hline \multirow[t]{2}{*}{ Variáveis } & \multicolumn{2}{|c|}{ Total } & \multicolumn{2}{|c|}{ Autoavaliação negativa } \\
\hline & $\mathbf{n}$ & $\%$ & $\mathrm{n}$ & $\%$ \\
\hline Total & 2.697 & 100,00 & 873 & 32,37 \\
\hline \multicolumn{5}{|l|}{ Raça/Cor } \\
\hline Branca & 1.171 & 43,42 & 325 & 27,75 \\
\hline Preta & 282 & 10,46 & 113 & 40,07 \\
\hline Parda & 1.244 & 46,13 & 435 & 34,97 \\
\hline \multicolumn{5}{|l|}{ Sexo } \\
\hline Masculino & 1.286 & 47,68 & 365 & 28,38 \\
\hline Feminino & 1.411 & 52,32 & 508 & 36,00 \\
\hline \multicolumn{5}{|l|}{ Renda (salários mínimos) } \\
\hline Até 2 & 953 & 35,34 & 415 & 43,55 \\
\hline $2-5$ & 1.016 & 37,67 & 292 & 28,74 \\
\hline $5-10$ & 436 & 16,17 & 105 & 24,08 \\
\hline 10 ou + & 292 & 10,83 & 61 & 20,89 \\
\hline \multicolumn{5}{|l|}{ Escolaridade } \\
\hline Fundamental & 1.608 & 59,62 & 690 & 42,91 \\
\hline Ensino médio & 764 & 28,33 & 143 & 18,72 \\
\hline Superior & 325 & 12,05 & 40 & 12,31 \\
\hline \multicolumn{5}{|l|}{ Estado civil } \\
\hline Solteiro & 901 & 33,41 & 206 & 22,86 \\
\hline Casado/Morando junto & 1.451 & 53,80 & 495 & 34,11 \\
\hline Separado/Divorciado & 182 & 6,75 & 81 & 44,51 \\
\hline Viúvo & 162 & 6,01 & 90 & 55,56 \\
\hline Ignorado & 1 & 0,04 & 1 & 100,00 \\
\hline \multicolumn{5}{|l|}{ Seguro saúde } \\
\hline Não & 1.988 & 73,71 & 714 & 35,92 \\
\hline Sim & 708 & 26,25 & 158 & 22,32 \\
\hline Ignorado & 1 & 0,04 & 1 & 100,00 \\
\hline \multicolumn{5}{|l|}{ Cadastro no PSF } \\
\hline Não & 1.580 & 58,58 & 459 & 29,05 \\
\hline Sim & 1.068 & 39,60 & 401 & 37,55 \\
\hline Ignorado & 49 & 1,82 & 13 & 26,53 \\
\hline
\end{tabular}

PSF: Programa Saúde da Família.

Foi realizado um teste de sensitividade para as categorias de saúde autoavaliada, onde a categoria "regular" foi incluída junto com as positivas. Para esse teste, o número de casos (indivíduos com saúde autoavaliada negativa) diminuiu consideravelmente, representando agora 5,8\% do total. Os resultados para modelo completo e os modelos com as características municipais e estaduais encontram-se na Tabela 4. Nos três modelos, pretos e pardos continuam a apresentar maior razão de chances de saúde autoavaliada que brancos, mas para essa categorização os resultados perderam significância estatística $(p>0,05)$.

\section{Discussão}

O presente estudo verificou que pretos e pardos apresentaram maior razão de chances de autoavaliarem sua situação de saúde como negativa, em relação a brancos. No caso da categorização original, esse resultado não perdeu significância estatística mesmo quando variáveis demográficas, socioeconômicas, de acesso a serviços de saúde, e características do local de residência (municipal e estadual) foram adicionadas ao modelo multinível.

No caso da inclusão da autoavaliação "regular" de saúde entre as positivas, o resultado não 
Tabela 2

Modelos multinível com a inclusão de variáveis demográficas, socioeconômicas e conjugal. Brasil, 2010.

\begin{tabular}{|c|c|c|c|c|c|c|c|c|c|c|}
\hline \multirow{2}{*}{ Variáveis (referência) } & \multicolumn{2}{|c|}{ Modelo nulo } & \multicolumn{2}{|c|}{ Modelo 1} & \multicolumn{2}{|c|}{ Modelo 2} & \multicolumn{2}{|c|}{ Modelo 3} & \multicolumn{2}{|c|}{ Modelo 4} \\
\hline & & & OR & IC95\% & OR & IC95\% & OR & IC95\% & OR & IC95\% \\
\hline \multicolumn{11}{|l|}{ Raça/Cor (branca) } \\
\hline Preta & & & 1,61 & $1,22-2,14$ & 1,91 & $1,39-2,61$ & 1,72 & $1,26-2,36$ & 1,75 & $1,27-2,41$ \\
\hline Parda & & & 1,32 & $1,10-1,60$ & 1,48 & $1,20-1,83$ & 1,39 & $1,12-1,72$ & 1,39 & $1,13-1,72$ \\
\hline Sexo (feminino vs. masculino) & & & & & 1,44 & $1,21-1,72$ & 1,42 & $1,18-1,70$ & 1,41 & $1,17-1,70$ \\
\hline Idade & & & & & 1,05 & $1,05-1,06$ & 1,05 & $1,04-1,05$ & 1,04 & $1,04-1,05$ \\
\hline \multicolumn{11}{|l|}{ Renda (até 2 salários mínimos) } \\
\hline $2-5$ & & & & & & & 0,69 & $0,56-0,85$ & 0,69 & $0,55-0,86$ \\
\hline $5-10$ & & & & & & & 0,73 & $0,54-0,98$ & 0,74 & $0,55-0,99$ \\
\hline $10 \mathrm{ou}+$ & & & & & & & 0,55 & $0,38-0,79$ & 0,54 & $0,38-0,78$ \\
\hline \multicolumn{11}{|l|}{ Escolaridade (Fundamental) } \\
\hline Ensino Médio & & & & & & & 0,60 & $0,48-0,76$ & 0,60 & $0,47-0,77$ \\
\hline Superior & & & & & & & 0,37 & $0,25-0,54$ & 0,37 & $0,25-0,55$ \\
\hline \multicolumn{11}{|l|}{ Estado civil (solteiro) } \\
\hline Casado/Morando junto & & & & & & & & & 1,14 & $0,91-1,41$ \\
\hline Separado/Divorciado & & & & & & & & & 1,30 & $0,89-1,90$ \\
\hline Viúvo & & & & & & & & & 1,03 & $0,68-1,57$ \\
\hline Variância ao nível estadual (DP) & 0,224 & $(0,107)$ & 0,193 & $(0,096)$ & 0,255 & $(0,120)$ & 0,202 & $(0,110)$ & 0,217 & $(0,114)$ \\
\hline Variância ao nível municipal (DP) & 0,027 & $(0,023)$ & 0,016 & $(0,019)$ & 0,045 & $(0,050)$ & 0,054 & $(0,065)$ & 0,060 & $(0,050)$ \\
\hline DIC & $3.339,21$ & & $3.331,58$ & & $2.978,38$ & & $2.918,16$ & & $2.917,08$ & \\
\hline
\end{tabular}

DIC: deviance information criterion; DP: desvio-padrão; IC95\%: intervalo de 95\% de confiança; OR: odds ratio.

foi estatisticamente significativo em nenhum dos modelos, apesar de manter a mesma direção estatística, ou seja, de pretos e pardos apresentarem maior razão de chances que brancos. Isso pode indicar a existência de um gradiente no efeito de raça/cor na saúde. Uma possibilidade é que raça/cor seja importante para explicar o aumento da proporção de autoavaliação regular em relação a bom, mas não necessariamente de ruim em relação a regular. Estudos que incluam uma categorização mais detalhadas, como uma escala de zero a dez, serão importante para identificar a presença de um gradiente de associação.

O uso de uma análise multinível permitiu verificar que características dos municípios e dos estados de residência não tiveram importância estatisticamente significativa para explicar a maior razão de chances verificada para pretos e pardos. Na categorização original, a variância do nível estadual foi inicialmente estatisticamente significativa, mas com a inclusão das variáveis individuais perdeu a significância. Nos modelos com a recategorização da autoavaliação regular, tanto a variância estadual quanto a municipal foram não significativas. A introdução de características do município e do estado de residência não alterou significativamente a razão de chances entre raça e autoavaliação de saúde em nenhuma das duas categorizações. A única variável referente ao local de residência que foi estatisticamente significativa (apesar de apenas marginalmente) foi a taxa de alfabetização para ambas as análises.

Os parâmetros das variáveis de controle incluídas nos modelos apresentaram a direção esperada. A razão de chances de autoavaliar a saúde como negativa foi significativamente maior para mulheres, indivíduos mais velhos, com menor renda, com menor educação e entre aqueles sem seguro privado de saúde para ambas as categorizações. Por outro lado, o cadastro do domicílio no PSF esteve associado a maior chance de autoavaliação negativa de saúde nas duas análises, apesar de não ter sido estatisticamente significativo. Isso pode ser consequência de confusão sobre o cadastro ou não no PSF por 
Modelos multinível com a inclusão de variáveis demográficas, socioeconômicas, de acesso a serviços de saúde e de características do local de residência. Brasil, 2010.

\begin{tabular}{|c|c|c|c|c|c|c|}
\hline \multirow[t]{2}{*}{ Variáveis (referência) } & \multicolumn{2}{|c|}{ Modelo 5} & \multicolumn{2}{|c|}{ Modelo 6} & \multicolumn{2}{|c|}{ Modelo 7} \\
\hline & OR & IC95\% & OR & IC95\% & OR & IC95\% \\
\hline \multicolumn{7}{|l|}{ Raça/Cor (branca) } \\
\hline Preta & 1,71 & $1,24-2,37$ & 1,71 & $1,23-2,36$ & 1,70 & $1,23-2,35$ \\
\hline Parda & 1,37 & $1,10-1,71$ & 1,35 & $1,09-1,67$ & 1,36 & $1,09-1,70$ \\
\hline Sexo (feminino vs. masculino) & 1,39 & $1,15-1,67$ & 1,43 & $1,19-1,72$ & 1,43 & $1,18-1,72$ \\
\hline Idade & 1,05 & $1,04-1,05$ & 1,05 & $1,04-1,05$ & 1,05 & $1,04-1,05$ \\
\hline \multicolumn{7}{|l|}{ Renda (até 2 salários mínimos) } \\
\hline $2-5$ & 0,71 & $0,57-0,88$ & 0,72 & $0,58-0,89$ & 0,72 & $0,59-0,89$ \\
\hline $5-10$ & 0,79 & $0,59-1,07$ & 0,79 & $0,59-1,07$ & 0,80 & $0,59-1,08$ \\
\hline $10 \mathrm{ou}+$ & 0,62 & $0,43-0,90$ & 0,59 & $0,41-0,86$ & 0,61 & $0,42-0,88$ \\
\hline \multicolumn{7}{|l|}{ Escolaridade (Fundamental) } \\
\hline Ensino Médio & 0,63 & $0,49-0,80$ & 0,63 & $0,50-0,80$ & 0,63 & $0,50-0,81$ \\
\hline Superior & 0,41 & $0,27-0,60$ & 0,41 & $0,28-0,61$ & 0,42 & $0,28-0,62$ \\
\hline Seguro saúde (não vs. sim) & 1,45 & $1,14-1,85$ & 1,44 & $1,14-1,81$ & 1,46 & $1,15-1,85$ \\
\hline Cadastro no PSF (não vs. sim) & 0,86 & $0,70-1,06$ & & & & \\
\hline \multicolumn{7}{|l|}{ Município } \\
\hline Percentual de pretos e pardos & & & 1,01 & $0,99-1,02$ & & \\
\hline Taxa de alfabetização & & & 0,98 & $0,96-0,99$ & & \\
\hline Renda nominal média & & & 1,00 & $1,00-1,00$ & & \\
\hline \multicolumn{7}{|l|}{ Estado } \\
\hline Percentual de pretos e pardos & & & & & 1,00 & $0,98-1,01$ \\
\hline Taxa de alfabetização & & & & & 0,97 & $0,94-0,99$ \\
\hline Renda nominal média & & & & & 1,00 & $1,00-1,00$ \\
\hline Variância ao nível estadual (DP) & 0,197 & $(0,110)$ & 0,169 & $(0,107)$ & 0,148 & $(0,092)$ \\
\hline Variância ao nível municipal (DP) & 0,027 & $(0,036)$ & 0,086 & $(0,054)$ & 0,014 & $(0,014)$ \\
\hline DIC & $2.861,39$ & & $2.904,77$ & & $2.905,86$ & \\
\hline
\end{tabular}

DIC: deviance information criterion; DP: desvio-padrão; IC95\%: intervalo de 95\% de confiança; OR: odds ratio;

PSF: Programa Saúde da Família.

parte dos indivíduos, ou ser devido a causalidade reversa, onde indivíduos mais pobres (e portanto com pior saúde) têm maior probabilidade de residirem em um domicílio cadastrado no PSF 34,35 .

O presente estudo tem algumas limitações importantes, sendo a principal delas a análise da autoavaliação de saúde, o que introduz uma possível barreira para a generalização dos resultados, já que pesquisas apontam para o efeito de diferenças linguísticas e culturais na resposta a essa questão 36 . Por exemplo, um estudo realizado nos Estados Unidos verificou que latinos apresentaram maior probabilidade de classificar a sua situação de saúde como ruim quando a pergunta foi feita em espanhol 37 .

Por outro lado, diversos estudos têm validado a associação entre autoavaliar sua saúde como negativa e maior probabilidade de óbito 16,17 .
Um estudo recente analisando idosos brasileiros, concluiu que a autoavaliação de saúde apresentou um poder preditivo para óbito semelhante a um escore composto por dez variáveis de saúde (incluindo pressão arterial, diabetes, índice de massa corpórea, entre outros) ${ }^{38}$. A autoavaliação negativa da saúde também tem sido associada a um maior risco de morbidades como diabetes e doença coronariana 39,40 .

Outra limitação do estudo é a ausência de critérios específicos para definir raça/cor ${ }^{41}$, o que tem particular importância em um país com altos níveis de miscigenação como o Brasil 42 . Segundo Muniz 11 (p. 282), raça "não é uma característica fixa e imutável e muito menos determinística", estando sujeita a variações temporais, espaciais e contextuais. Para a presente análise foi utilizada a autodeterminação étnico-racial, que é considerada o padrão ouro em estudos da área 43 . Apesar 
Modelos multinível com a inclusão da autoavaliação de saúde regular entre as categorias positivas. Brasil, 2010.

\begin{tabular}{|c|c|c|c|c|c|c|}
\hline \multirow[t]{2}{*}{ Variáveis (referência) } & \multicolumn{2}{|c|}{ Modelo 1} & \multicolumn{2}{|c|}{ Modelo 2} & \multicolumn{2}{|c|}{ Modelo 3} \\
\hline & OR & IC95\% & OR & IC95\% & OR & IC95\% \\
\hline \multicolumn{7}{|l|}{ Raça/Cor (branca) } \\
\hline Preta & 1,33 & $0,76-2,33$ & 1,32 & $0,74-2,38$ & 1,28 & $0,73-2,27$ \\
\hline Parda & 1,03 & $0,70-1,51$ & 1,01 & $0,65-1,57$ & 1,01 & $0,67-1,53$ \\
\hline Sexo (feminino vs. masculino) & 1,51 & $1,05-2,17$ & 1,54 & $1,08-2,19$ & 1,55 & $1,09-2,20$ \\
\hline Idade & 1,04 & $1,03-1,05$ & 1,04 & $1,03-1,05$ & 1,04 & $1,03-1,06$ \\
\hline \multicolumn{7}{|l|}{ Renda (até 2 salários mínimos) } \\
\hline $2-5$ & 0,53 & $0,35-0,80$ & 0,53 & $0,35-0,81$ & 0,55 & $0,38-0,81$ \\
\hline $5-10$ & 0,64 & $0,35-1,18$ & 0,65 & $(0,35-1,20$ & 0,65 & $0,35-1,23$ \\
\hline 10 ou + & 0,69 & $0,34-1,38$ & 0,67 & $0,33-1,35$ & 0,70 & $0,35-1,39$ \\
\hline \multicolumn{7}{|l|}{ Escolaridade (Fundamental) } \\
\hline Ensino Médio & 0,55 & $0,32-0,97$ & 0,59 & $0,33-1,04$ & 0,57 & $1,02-0,32$ \\
\hline Ensino Superior & 0,27 & $0,08-0,87$ & 0,29 & $0,10-0,85$ & 0,28 & $0,09-0,91$ \\
\hline Seguro saúde (não vs. sim) & 1,39 & $0,84-2,29$ & 1,28 & $0,81-1,99$ & 1,45 & $0,88-2,41$ \\
\hline Cadastro no PSF (não vs. sim) & 0,80 & $0,56-1,15$ & 0,89 & $0,61-1,29$ & 0,80 & $0,56-1,16$ \\
\hline \multicolumn{7}{|l|}{ Município } \\
\hline Percentual de pretos e pardos & & & 1,00 & $0,98-1,01$ & & \\
\hline Taxa de alfabetização & & & 0,97 & $0,94-0,99$ & & \\
\hline Renda nominal média & & & 1,00 & $1,00-1,00$ & & \\
\hline \multicolumn{7}{|l|}{ Estado } \\
\hline Percentual de pretos e pardos & & & & & 1,00 & $1,00-1,01$ \\
\hline Taxa de alfabetização & & & & & 1,00 & $0,97-1,02$ \\
\hline Renda nominal média & & & & & 1,00 & $1,00-1,00$ \\
\hline Variância ao nível estadual (DP) & 0,150 & $(0,184)$ & 0,280 & $(0,218)$ & 0,329 & $(0,262)$ \\
\hline Variância ao nível municipal (DP) & 0,150 & $(0,202)$ & 0,115 & $(0,122)$ & 0,021 & $(0,025)$ \\
\hline DIC & $1.057,15$ & & 1057,73 & & $1.054,60$ & \\
\hline
\end{tabular}

DIC: deviance information criterion; DP: desvio-padrão; IC95\%: intervalo de 95\% de confiança; OR: odds ratio;

PSF: Programa Saúde da Família.

disso, a análise dos resultados não está livre de alguma variação contextual. Um estudo realizado por Bastos et al. ${ }^{44}$, por exemplo, verificou que indivíduos apresentaram menor probabilidade de se identificarem como pretos quando questionados por entrevistadoras pretas.

Apesar de o Brasil possuir a maior população negra fora da África 45 , o número de estudos epidemiológicos que analisaram a questão étnicoracial ainda é escasso, mesmo com a teoria de democracia racial tendo caído em descrédito nas últimas décadas 2. Para Araújo et al. ${ }^{4}$, isso pode ser uma consequência da baixa presença de pretos e pardos em cargos em situação de poder e influência na determinação de políticas públicas. Segundo Lopes 46, a questão da multiplicidade de fatores socioeconômicos associados à questão racial pode fazer com que muitas análises não encontrem associação estatisticamente significativa entre saúde e raça/cor, o que diminuiria o número de artigos publicados. Outro fator importante tem sido a ausência de uma questão sobre raça/cor em pesquisas e registros governamentais até recentemente. Apesar do baixo número de publicações, estudos recentes têm apontado características étnico-raciais como um marcador de risco para determinadas condições de saúde 10 .

O fato de a associação entre raça/cor e autoavaliação de saúde não ter sido estatisticamente significativa quando a categoria "regular" foi incluída entre as positivas pode indicar a presença de um gradiente no efeito das características étnico-raciais em saúde. Por outro lado, não se pode descartar limitações inerentes a estudos étnico-racial como as diferenças idiossincráticas na autodeterminação racial e na percepção de racismo 47, como explicativas da associação estatisticamente significativa verificada na categorização original. 
As complexidades das pesquisas étnico-raciais na área de saúde foram também apontadas por um estudo recente de Macinko et al. ${ }^{48}$, que, ao analisarem o resultado de uma pesquisa de 12.930 residentes da Região Metropolitana de Belo Horizonte, Minas Gerais, verificaram que a probabilidade prevista de declarar ter sido vítima de discriminação foi maior para homens e mulheres com saúde ruim que se autodeclararam com pretos ( $31 \%$ e $35 \%$, respectivamente). Entretanto, segundo os autores, raça/cor foi apenas uma das variáveis associadas com discriminação, o que pode indicar a complexidade da relação entre raça/cor, discriminação e saúde.

\section{Resumen}

Las revisiones bibliográficas recientes muestran que los estudios que analizan las disparidades etno-raciales en Brasil siguen siendo escasos. Es necesario un enfoque multinivel, debido a que el concepto de raza es una construcción social, y es susceptible de variar según la zona de residencia. El presente análisis incluyó a 2.697 personas de 145 municipios brasileños, separados por raza (blanco, negro y mestizos). Se ajustaron modelos multinivel, usando la inferencia bayesiana con métodos Markov Chain Monte Carlo. Después de incluir las variables demográficas, socioeconómicas y de acceso a la salud como controles, las personas identificadas como negros y mestizos tuvieron un odds ratio más elevado al juzgar su salud en términos negativos $(O R=1,71$; IC95\%: 1,24; 2,37 y OR = 1,37; 95\%CI: 1,10; 1,71, respectivamente). Después de la recategorización de la variable dependiente, las disparidades etno-raciales perdieron significación estadística. Los resultados indican que las disparidades etno-raciales en salud en Brasil pueden ser más complejas y profundas de lo esperado.

Origen Étnico y Salud; Análisis Multinivel; Factores Socieconómicos
O Brasil é um país com amplas e históricas desigualdades. Apesar de melhorias, seu coeficiente de Gini, utilizado para medir desigualdade de renda, ainda encontra-se entre os mais altos do mundo (World Bank. Gini index. http://data. worldbank.org/indicator/SI.POV.GINI, acessado em 10/Jan/2012). Essas desigualdades, por sua vez, se refletem em um grande número de problemas sociais e de saúde 49 . A introdução da análise das diferenças étnico-raciais pode ajudar a não só compreender melhor a origem de algumas dessas disparidades, mas também a identificar possibilidades de atuação para diminuí-las.

\section{Colaboradores}

A. D. P. Chiavegatto Filho participou da redação do artigo, interpretação e análise dos dados, revisão crítica do artigo e aprovação final da versão publicada. R. Laurenti contribuiu significativamente na interpretação e análise dos dados, revisão crítica do artigo e aprovação final da versão publicada.

\section{Agradecimentos}

A FAPESP (processo 2012/09717-2) pelo financiamento. 


\section{Referências}

1. Satcher D, Fryer Jr. GE, McCann J, Troutman A, Woolf SH, Rust G. What if we were equal? A comparison of the black-white mortality gap in 1960 and 2000. Health Affair 2005; 24:459-64.

2. Chor D, Lima CRA. Aspectos epidemiológicos das desigualdades raciais em saúde no Brasil. Cad Saúde Pública 2005; 21:1586-94.

3. Travassos C, Williams DR. The concept and measurement of race and their relationship to public health: a review focused on Brazil and the United States. Cad Saúde Pública 2004; 20:660-78.

4. Araújo EM, Costa MCN, Hogan VK, Araújo TM, Dias AB, Oliveira LOA, et al. A utilização da variável raça/cor em Saúde Pública. Interface Comum Saúde Educ 2009; 13:383-94.

5. Bailey SR. Legacies of race: identities, attitudes, and politics in Brazil. Stanford: Stanford University Press; 2009.

6. Daniel GR. Race and multiraciality in Brazil and the United States: converging paths? University Park: Pennsylvania State University Press; 2006.

7. Telles EE. Race in another America: the significance of skin color in Brazil. Princeton: Princeton University Press; 2004

8. Araújo EM, Costa MCN, Noronha CV, Hogan VK, Vines AI, Araújo TM. Desigualdades em saúde e raça/cor da pele: revisão da literatura do Brasil e dos Estados Unidos (1996-2005). Saúde Coletiva 2010; 40:116-21

9. Monteiro S, Sansone L. Desigualdade em saúde, raça e etnicidade: questões e desafios. In: Monteiro S, Sansone L, organizadores. Etnicidade na América Latina: um debate sobre raça, saúde e direitos reprodutivos. Rio de Janeiro: Editora Fiocruz; 2004. p. $45-56$.

10. Kabad JF, Bastos JL, Santos RV. Raça, cor e etnia em estudos epidemiológicos sobre populações brasileiras: revisão sistemática na base PubMed. Physis (Rio J.) 2012; 22:895-918.

11. Muniz JO. Sobre o uso da variável raça-cor em estudos quantitativos. Revista de Sociologia e Política $2010 ; 18: 277-91$

12. Bastos JL, Peres MA, Peres KG, Dumith SC, Gigante DP. Diferenças socioeconômicas entre autoclassificação e heteroclassificação de cor/raça. Rev Saúde Pública 2008; 42:324-34.

13. Teixeira NZF, Pereira WR, Barbosa DA, Vianna LAC. Mortalidade materna e sua interface com a raça em Mato Grosso. Rev Bras Saúde Matern Infant 2012; 12:27-35.

14. Cardoso AM, Santos RV, Coimbra Jr. CE. Mortalidade infantil segundo raça/cor no Brasil: o que dizem os sistemas nacionais de informação? Cad Saúde Pública 2005; 21:1602-8.

15. Bairros FS, Meneghel SN, Dias-da-Costa JS, Bassani DG, Menezes AMB, Gigante DP, et al. Racial inequalities in access to women's health care in southern Brazil. Cad Saúde Pública 2011; 27:2364-72.

16. Burström B, Fredlund P. Self-rated health: Is it as good a predictor of subsequent mortality among adults in lower as well as in higher social classes? J Epidemiol Community Health 2001; 55:836-40.
17. Siegel M, Bradley EH, Kasl SV. Self-rated life expectancy as a predictor of mortality: evidence from the HRS and AHEAD surveys. Gerontology 2003; 49:265-71.

18. Rodrigues CG, Maia AG. Como a posição social influencia a auto-avaliação do estado de saúde? Uma análise comparativa entre 1998 e 2003. Cad Saúde Pública 2010; 26:762-74.

19. Dachs JNW, Norberto W. Determinantes das desigualdades na auto-avaliação do estado de saúde no Brasil: análise dos dados da PNAD/1998. Ciênc Saúde Coletiva 2002; 7:641-57.

20. Barata RB, Almeida MF, Montero CV, Silva ZP. Health inequalities based on ethnicity in individuals aged 15 to 64, Brazil, 1998. Cad Saúde Pública 2007; 23:305-13.

21. Santos SM, Chor D, Werneck GL, Coutinho ESE Associação entre fatores contextuais e auto-avaliação de saúde: uma revisão sistemática de estudos multinível. Cad Saúde Pública 2007; 23:2533-54.

22. Bécares L, Nazroo J, Albor C, Chandola T, Stafford M. Examining the differential association between self-rated health and area deprivation among white British and ethnic minority people in England. Soc Sci Med 2011; 64:616-24.

23. Shaw RJ, Pickett KE. The association between ethnic density and poor self-rated health among US Black and Hispanic people. Ethn Health 2011; 16:225-44.

24. Osorio RG. O sistema classificatório de "cor ou raça” do IBGE. Brasília: Instituto de Pesquisa Econômica Aplicada; 2003.

25. Instituto de Pesquisa Econômica Aplicada. SIPS Sistema de Indicadores de Percepção Social: metodologia http://www.ipea.gov.br/portal/images/ stories/PDFs/SIPS/120418_sips_metodologia.pdf (acessado em 10/Jan/2012)

26. Instituto de Pesquisa Econômica Aplicada. Sistema de Indicadores de Percepção Social: saúde. Brasília: Instituto de Pesquisa Econômica Aplicada; 2012.

27. Barros MVG, Arkus VN. Comportamentos de risco, auto-avaliação do nível de saúde e percepção de estresse entre trabalhadores da indústria. Rev Saúde Pública 2001; 35:554-64.

28. DeSalvo KB, Bloser N, Reynolds K, He J, Muntner P. Mortality prediction with a single general selfrated health question. J Gen Intern Med 2005; 21 : 267-75.

29. Diez-Roux AV. Multilevel analysis in public health research. Annu Rev Public Health 2000; 21:171-92.

30. Leyland AH, Groenewegen PP. Multilevel modelling and public health policy. Scand J Public Health 2003; 31:267-74.

31. Robinson WS. Ecological correlations and the behavior of individuals. Int J Epidemiol 2009; 38 . 337-41.

32. Rodriguez G, Goldman N. An assessment of estimation procedures for multilevel models with binary responses. J R Stat Soc Series A Stat Soc 1995; 158:73-89. 
33. Subramanian S, Jones K, Kaddour A, Krieger N. Revisiting Robinson: the perils of individualistic and ecologic fallacy. Int J Epidemiol 2009; 38:342-60.

34. Escorel S, Giovanella L, Mendonça M, Senna MCM. O Programa de Saúde da Família e a construção de um novo modelo para a atenção básica no Brasil. Rev Panam Salud Pública 2007; 21:164-76.

35. Goldbaum M, Gianini RJ, Novaes HMD, César CLG. Utilização de serviços de saúde em áreas cobertas pelo programa saúde da família (Qualis) no Município de São Paulo. Rev Saúde Pública 2005; 39:90-9.

36. Lima-Costa MF, Steptoe A, Cesar CC, Oliveira C, Proietti FA, Marmot M. The influence of socioeconomic status on the predictive power of self-rated health for 6-year mortality in English and Brazilian older adults: the ELSA and Bambuí cohort studies. Ann Epidemiol 2012; 22:644-8.

37. Viruell-Fuentes EA, Morenoff JD, Williams DR, House JS. Language of interview, self-rated health and the other Latino health puzzle. Am J Public Health 2011; 101:1306-13.

38. Lima-Costa M, Cesar C, Chor D, Proietti F. Selfrated health compared with objectively measured health status as a tool for mortality risk screen ing in older adults: 10-year follow-up of the Bambuí Cohort Study of Aging. Am J Epidemiol 2012; 175:228-35.

39. Latham K, Peek CW. Self-rated health and morbidity onset among late midlife US adults. J Geronto B Psychol Sci Soc Sci 2013; 68:107-16.

40. Møller L, Kristensen TS, Hollnagel H. Self-rated health as a predictor of coronary heart disease in Copenhagen, Denmark. J Epidemiol Community Health 1996; 50:423-8.
41. Laguardia J. O uso da variável "raça" na pesquisa em saúde. Physis (Rio J.) 2004; 14:197-234.

42. Bhopal RS. Ethnicity, race, and health in multicultural societies: foundations for better epidemiology, public health, and health care. New York: Oxford University Press; 2007.

43. Boehmer U, Kressin NR, Berlowitz DR, Christiansen CL, Kazis LE, Jones JA. Self-reported vs. admin istrative race/ethnicity data and study results. Am J Public Health 2002; 92:1471-2.

44. Bastos JL, Dumith SC, Santos RV, Barros AJD, Del Duca GF, Gonçalves H, et al. Does the way I see you affect the way I see myself? Associations between interviewers' and interviewees' “color/ race” in southern Brazil. Cad Saúde Pública 2009; 25:2111-24

45. Silva NV. Cor e mobilidade ocupacional. In: Silva NV, Pastore J, organizadores. Mobilidade social no Brasil. São Paulo: Makron Books; 2000. p. 85-98.

46. Lopes F. Para além da barreira dos números: desigualdades raciais e saúde. Cad Saúde Pública 2005; 21:1595-601.

47. Sacramento AM, Nascimento ER. Racismo e saúde: representações sociais de mulheres e profissionais sobre o quesito cor/raça. Rev Esc Enferm USP 2011; 45:1142-9.

48. Macinko J, Mullachery P, Proietti FA, Lima-Costa MF. Who experiences discrimination in Brazil? Evidence from a large metropolitan region. Int J Equity Health 2012; 11:80.

49. Chiavegatto Filho ADP, Gotlieb SLD, Kawachi I. Cause-specific mortality and income inequality in São Paulo, Brazil. Rev Saúde Pública 2012; 46:712-8.

Recebido em 19/Set/2012

Versão final reapresentada em 05/Mar/2013

Aprovado em 11/Mar/2013 